\title{
Expression of key components of the RNAi machinery are suppressed in Apis mellifera that suffer a high virus infection
}

\author{
Lina DE SMET ${ }^{1 *}$, Jorgen RAVOET ${ }^{1 *}$, Tom WENSELEERS ${ }^{2}$ and Dirk C. DE GRAAF ${ }^{1}$ \\ ${ }^{1}$ Laboratory of Molecular Entomology and Bee Pathology, Ghent University, Ghent, Belgium and ${ }^{2}$ Laboratory of Socioecology and \\ Social Evolution, University of Leuven, Leuven, Belgium
}

\begin{abstract}
Viruses are one of the major threats for honeybees and until now more than 20 different viruses have been discovered. Viruses and their hosts are engaged in a continuous arms race in which viral defense mechanisms drive the adaptive evolution of host immune genes, which in turn results in counter-adaptations of the viral immune antagonists. The honeybee immune responses to non-viral pathogens have been extensively studied, but little is known about the antiviral responses. Recent evidence suggests that the main mechanism of antiviral defense in insects is the RNA interference (RNAi) pathway. Furthermore, there is evidence that some viruses suppress this RNAi pathway in order to evade antiviral immunity. In the present study, we test this hypothesis by comparing the gene expression levels of some key components of the RNAi response of honeybees that were naturally infected with at least five viruses with those who were infected with only two or three viruses using a colorimetric microarray developed in-house, called BeeClinic, and subsequently confirmed by quantitative reverse-transcription-polymerase chain reaction (qRT-PCR). Our results show that key components of the RNAi pathway are indeed downregulated in highly infected bees. We were able to show that high virus loads suppress key RNAi components, which results in a counteraction of the host RNAi antiviral defense. As the RNAi is a primary defense against viruses, these findings shed new light on pathogen-host interactions and can help mitigate escalating colony losses worldwide.
\end{abstract}

Key words: antiviral, BeeClinic, colorimetric microarray, Dicer, gene expression, honeybee.

\section{INTRODUCTION}

Honeybees are exposed to a wide array of viruses (Chen \& Siede 2007; Runckel etal. 2011; Li etal. 2014; McMenamin \& Genersch 2015). Like all insects they lack an adaptive immune system and thus, the immune defense relies solely on the innate immune response, which is based on a constitutively active cellular and an inducible humoral immune response (Evans et al. 2006). Based on the honeybee genome, homologue members of the complex humoral immune response could be identified (Evans etal. 2006). Insects are able to trigger various

Correspondence: Lina De Smet, Laboratory of Molecular Entomology and Bee Pathology, Ghent University, Krijgslaan 218 / S2, 9000 Ghent, Belgium.

Email: lina.desmet@ugent.be

"Both authors contributed equally to this work.

Received 3 March 2016; accepted 26 May 2016; first published 13 September 2016. defense pathways depending on the type of infecting pathogen, and most of these pathways are interconnected. For fungal and bacterial infections, the Toll, Imd and Jak/STAT pathways have been implicated (Lemaitre \& Hoffmann 2007). Although these pathways also play a role in the clearance of viral infections their antiviral function seems to be virus-specific rather than being a generic antiviral response (Kemp \& Imler 2009). The interaction of the virus with the host's innate immune system plays a critical role in the outcome of the infection. The major mechanism of antiviral defense is the RNA interference (RNAi) pathway (Ding 2010). Most honeybee viruses are positive-sense single-stranded RNA (+ssRNA) viruses, which produce double-stranded RNA (dsRNA) replication intermediates (Chen 2011). This virus-generated dsRNA serves as a template for the RNAi machinery and produces small, interfering RNAs (siRNAs) that function to target viral RNA for degradation and hence inhibit replication (Ding 2010). Direct evidence of the antiviral role of RNAi insects comes 
from studies in Drosophila melanogaster, Aedes aegypti and Anopheles gambiae (Campbell et al. 2008; Saleh et al. 2009; Blair 2011). Recent studies in Apis mellifera and Apis cerana demonstrated the role of RNAi antiviral immunity in honeybees (Maori etal. 2009; Liu etal. 2010; Desai etal. 2012). In general, RNAi-based degradation of dsRNA involves the production of small non-coding RNAs, and their biogenesis and function are based on two proteins: Dicer (Dcr) and Argonaute (Ago) (Elbashir etal. 2001; Hammond et al. 2001). Their genes are strongly conserved in a wide range of species. However, as a result of evolutionary and immune adaptation processes, there are several paralogues of both proteins. The RNAi response is conserved in every insect model tested until now (Karlikow etal. 2014). The role of the RNAi response as an immune system combating viruses in bees is scarcely studied although recently its involvement in bumblebees has been shown (Niu etal. 2015; Piot et al. 2015). The inducer signal for a systemic response remains unknown. Nevertheless, evidence points to a signal of RNA nature (dsRNA, small RNA or RNP).

The honeybee immune responses to non-viral pathogens have been extensively studied (Chan etal. 2009; Schwarz \& Evans 2013), but far less is known about the immune system's antiviral responses. Recently, it has been shown that workers from collapsed honeybee colonies display an RNAi response. High-throughput sequencing and data analysis of small RNA from these bees showed a high number of reads of small RNA of 21-22 nt perfectly matching the Israeli acute paralysis virus (IAPV), Kashmir bee virus (KBV) and deformed wing virus (DWV) genomes in these colonies (Flenniken \& Andino 2013; Chejanovsky et al. 2014).

RNAi has also been associated with controlling the persistence of RNA virus infections in Drosophila (Goic et al. 2013). In honeybees, severe Varroa-mite infestations are often associated with persistent DWV infection (de Miranda etal. 2012). This hematophagous mite acts as vector for many viruses, but overt DWV infections in the pupal stage are very common. In such pupae, virusspecific siRNAs (mostly derived from DWV and $22 \mathrm{nt}$ in length) could be identified, but the virus-specific siRNA levels were not always proportional to the level of viral genomic RNA (Ryabov et al. 2014). This supports the hypothesis that suppression of the antiviral response may also exist. The detection of virus-specific siRNAs in several studies suggests a normal functioning of Dicer in honeybees (Desai etal. 2012). However, transcriptome studies studying the honeybee response to viral infections could not show significant changes in gene expression of the key components of the RNAi response, such as $d c r$ and ago (Ryabov etal. 2014). In Drosophila, cricket paralysis virus (CrPV) has been found to encode a potent suppressor that mutes the RNAi antiviral response (Nayak et al. 2010). This suppressor is located upstream of a highly conserved sequence (DVEXNPGP) within the $\mathrm{N}$-terminal regions of $\mathrm{CrPV}$ open reading frame- 1 (ORF-1) (Bennasser etal. 2006). This conserved motif (DIEENPGP) is also identified in the $\mathrm{N}$-terminal region of ORF-1 of IAPV and other members of the Dicistroviridae family infecting honeybees such as KBV and acute bee paralysis virus (ABPV) (Chen etal. 2014). Silencing of this putative immune suppressive protein led to significant reduction in IAPV replication, which suggests that IAPV may encode an RNAi suppressor (Chen etal. 2014). Viral suppressors of RNAi (VSR) proteins are often encoded in overlapping reading frames or suppressor activity will have evolved in unrelated viral proteins with other functions ( $\mathrm{Li} \&$ Ding 2006). As a result, viral suppressors differ greatly with respect to sequence, structure, and mode of action. The best studied VSR proteins are RNA-binding proteins that shield dsRNA produced during viral infection from Dicer processing and RNA-induced silencing complex (RISC) assembly (O'Neal etal. 2014). Other VSR proteins do not bind dsRNA or siRNAs, but exert an inhibitory effect on RNA silencing through a direct interaction with Ago2 (O’Neal et al. 2014). It was also shown that Dicer, Drosha and Ago 2 mRNA and protein expression levels were downregulated in mammalian cells infected with influenza $A$ virus, dengue virus and hepatitis $B$, which suggests that Drosha, Dicer and Ago2 regulate viral replication (Chinnappan etal. 2014). In this study, we compared the gene expression levels of some key components of the RNAi response of naturally infected honeybees using a colorimetric microarray fabricated in-house. In particular, we compared the expression level of bees that were naturally infected with at least five viruses with those that were infected with two or three viruses. Our prediction was that that the RNAi response would change with a higher virus load.

\section{MATERIALS AND METHODS}

\section{Honeybee samples}

Honeybee samples with low and high virus loads were selected from previous studies (De Smet etal. 2012; Ravoet etal. 2013). All the bees were sampled at the bee hive entrance, which means that they are at least four weeks old. Bees with a low virus load were infected by two or three viruses while those with a high virus load were infected with at least five viruses. Deformed wing virus and bee macula like virus were retrieved in almost all samples. The exact virus load for the different selected samples is given in Table S1. Eight colonies for each 
condition were selected, from which three bees were analyzed using the BeeClinic microarray. For the quantitative reverse-transcription-polymerase chain reaction (qRT-PCR) validation experiment the same bee samples were used.

\section{Preparation of total RNA}

Total RNA was isolated using the RNeasy lipid tissue mini kit (Qiagen, Hilden, Germany) starting from one complete honeybee. The tissues were homogenized by mechanical agitation in a TissueLyser (Precellys, PEQLAB Biotechnology, Germany) for $90 \mathrm{~s}$ at $30 \mathrm{~Hz}$ in the presence of a pair stainless steel beads and $1 \mathrm{~mL}$ Qiazol (Qiagen) lysis reagent. The total RNA was isolated according to the recommendations of the manufacturer's protocol, eluting the RNA in a final volume of $50 \mu \mathrm{L}$. The concentration of the total RNA was measured using a Nanodrop (Isogen; Nippon Gene, Tokyo, Japan).

\section{Design of colorimetric microarray}

The principle of the colorimetric hybridization assay is outlined in Figure 1. The sample, which contains digoxigenin (DIG)-labeled cDNA, is used for hybridization. The hybridization signals are developed using anti-DIG antibodies (Roche Applied Science, Indianapolis, IN, USA), which are alkaline phosphatase (AP) conjugated. The AP system enables a colorimetric detection of hybridization: the immobilized enzyme's reaction with its substrate (BCIP/NBT) generates a purple precipitate on hybridized spots. The stained microarrays are finally imaged using a high-resolution flatbed scanner and the grayscale image is analyzed.

\section{A}

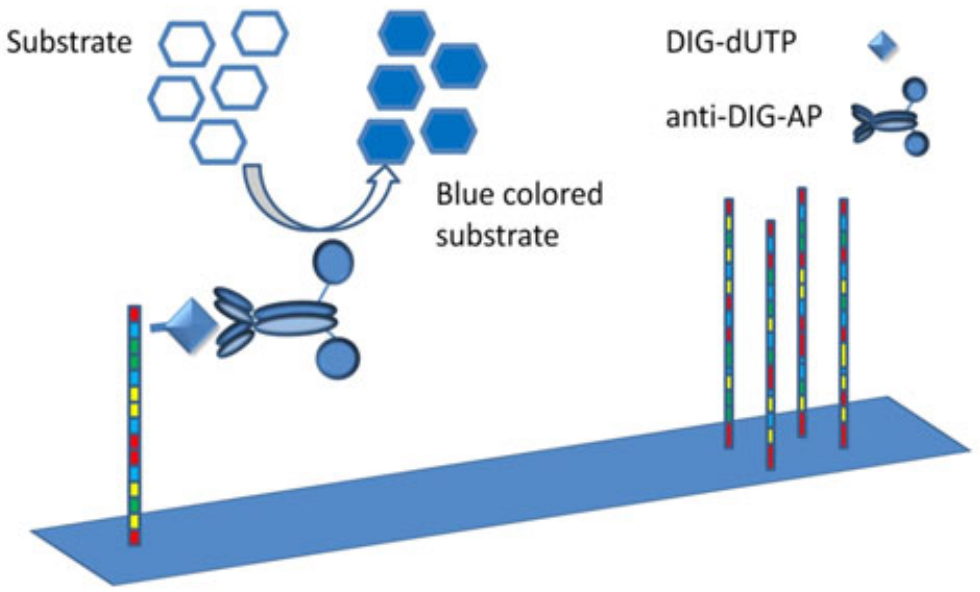

B

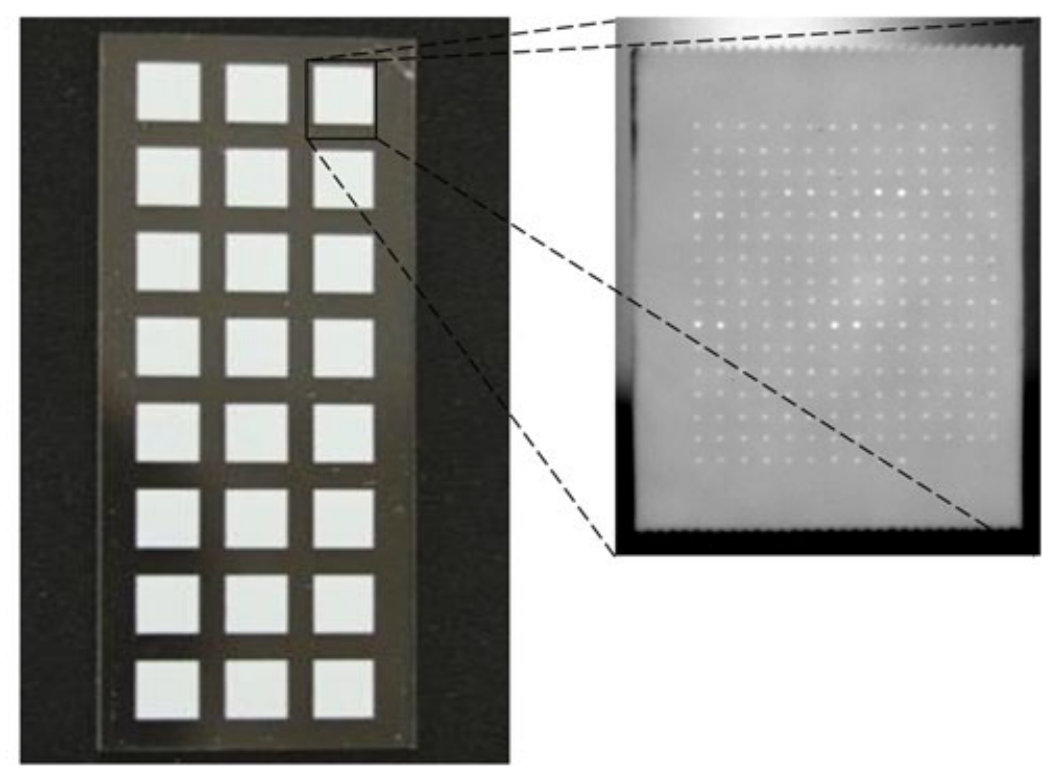

Figure 1 (A) General outline of the inhouse microarray procedure: 110 targets are printed per microarray. After hybridization of the digoxigenin (DIG)-labeled cDNA, an antidigoxigenin antibody-alkaline phosphatase conjugate is bound to the hybridized probe. The signal is detected with the colorimetric alkaline phosphatase substrate, NBT and BCIP. (B) 24-plex microarray slide and closeup of one field after development. 


\section{Targeted genes}

Our array targeted immunity genes that were previously included in the Beepath quantitative PCR array (Evans 2006) and was further extended with selected pathogen probes and markers for Varroa infestation (Alaux etal. 2011), Nosema infection (Dussaubat etal. 2012), nutritional stress (Alaux etal. 2011) and genes involved in ageing and in the RNAi machinery. For some threats, genome-wide transcriptome studies were available (Alaux etal. 2011; Dussaubat etal. 2012). For each threat, the five most up- and downregulated marker genes were selected from these studies. Based on quantitative trait locus (QTL) analysis, two genes for Varroa resistance (Behrens etal. 2011) and one gene for Nosema resistance (Huang etal. 2012) were also selected and included in the target list. Genes implied in ageing or in the RNAi machinery were selected based on literature data and homology (reviewed by Karlikow etal. 2014) with known Drosophila genes involved in the RNAi response (McMenamin \& Genersch 2015). This resulted in a total final set of 109 targets included on our array (Table S2). The corresponding oligonucleotides from the selected targets were used from the study of Johnson etal. (2009). New oligonucleotides were designed using the AlleleID7 (Premier Biosoft International; Palo Alto, CA, USA). All the probes were synthesized and desalted by Integrated DNA Technologies (Coralville, IA, USA). The probes were printed in duplicate on nitrocellulose coated glass slides by ArrayIT (Sunnyvale, CA, USA). Each slide is composed of 24 identical arrays, with each array harboring 109 targets in duplicate. The printed arrays were stored at room temperature until use.

\section{Labeling cDNA}

The cDNA was labeled with DIG using the Superscript Direct cDNA labeling system from Invitrogen (Carlsbad, CA, USA). Briefly, $25 \mu \mathrm{g}$ of total RNA was reverse transcribed into cDNA with anchored oligo(dT)20 primers. The reverse transcription procedure was used as described by the manufacturer, except that deoxynucleoside triphosphate (dNTP) was replaced by $2 \mathrm{mM}$ dATP, dGTP and dCTP, $1.3 \mathrm{mM}$ dTTP and $0.7 \mathrm{mM}$ alkali stable DIG-dUTP (Roche). Once the reverse transcription was completed, the enzyme was inactivated and the original RNA was degraded by alkaline hydrolysis. The labeled cDNA was purified using the Superscript III Direct Purification Module (Invitrogen) according to the kit instructions. Purified single-strand cDNA was eluted from the columns in $70 \mu \mathrm{L}$ DEPCtreated water.

\section{Hybridization and development}

Before hybridization, the microarrays are washed three times in BlockIT solution (ArrayIT) to remove unbound nucleotides, followed by a prehybridization step with BlockIT solution for $1 \mathrm{~h}$ at room temperature. After blocking, the array was washed three times for $5 \mathrm{~min}$ with $5 \times \operatorname{SSC}(0.15 \mathrm{M} \mathrm{NaCl}, 0.015 \mathrm{M}$ sodium citrate for $1 \times \mathrm{SSC})$ and $0.1 \%$ sodium dodecyl sulfate and mounted in a 24 well hybridization cassette from ArrayIT. Labeled cDNA corresponding with $3 \mu \mathrm{g}$ total RNA was mixed with hybridization buffer (final concentration $25 \%$ formamide, $5 \times$ SSC and $0.1 \%$ SDS) to a final volume of $75 \mu \mathrm{L}$ and heated for $1 \mathrm{~min}$ at $65^{\circ} \mathrm{C}$ to denature the cDNA and snap-cooled on ice for $30 \mathrm{~s}$. The labeled cDNA was then transferred to the array and hybridized overnight at $33.5^{\circ} \mathrm{C}$. After hybridization, the arrays were unmounted from the cassette and washed twice at $45^{\circ} \mathrm{C}$ for $5 \mathrm{~min}$ with $2 \times$ SSC and $0.1 \%$ SDS, twice with $0.5 \times$ SSC and $0.1 \%$ SDS at $45^{\circ} \mathrm{C}$ for $5 \mathrm{~min}$ followed by a $5 \mathrm{~min}$ wash at room temperature with $0.5 \times$ SSC.

Colorimetric detection is a three-step process. In the first step, membranes were treated with $1 \%$ blocking solution (Roche) for $30 \mathrm{~min}$ to prevent nonspecific attraction of the antibody to the membrane. In the following step the membranes were incubated with $1500 \times$ diluted anti-digoxigenin (Roche) in 1\% blocking solution. The antibodies are conjugated to alkaline phosphatase, which made colorimetric development possible. The unbound antibody was washed away in two washing steps for 15 min with PBS and the slides were equilibrated in TBS buffer for $5 \mathrm{~min}$. In the last step, the membrane carrying the hybridized probed and bound antibody conjugate was reacted with the colorimetric detection reagents nitro blue tetrazolium salt (NBT) and 5-bromo-4-chloro-3-indolyl phosphate (BCIP). The NBT/BCIP stock solution (Roche) contained $18.75 \mathrm{mg} /$ $\mathrm{mL}$ nitro blue tetrazolium chloride and $9.4 \mathrm{mg} / \mathrm{mL}$ BCIP, toluidine-salt in $67 \%$ DMSO $(\mathrm{v} / \mathrm{v})$. The slides were incubated in $15 \mathrm{~mL}$ TBS buffer containing $300 \mu \mathrm{L}$ NBT/BCIP for $30 \mathrm{~min}$. The reaction was stopped by washing the slides in distilled water. The array was dried by centrifugation. Subsequently, the slides were scanned using the ArrayIT SpotWareTM colorimetric microarray scanner at 16-bit grayscale depth and $5 \mu \mathrm{m}$ resolution and saved in TIFF format.

\section{Data analysis}

The TIFF images were processed with Mapix (Innopsys, France) to ascribe a value to the spot intensity, which was corrected by background intensity. The intensity data were standardized across different samples using the 
control reference genes RPL8 and actin. To test for differential expression, the Bayesian adjusted $t$-statistics from the linear models for Micoarray data (limma) package were used (Smyth 2004; Ritchie etal. 2015). The adjusted $P$-value was calculated using the method developed by Benjamin and Hochberg (Evans \& Spivak 2010).

\section{Validation by qRT-PCR}

Using random hexamer primers, $2 \mu \mathrm{g}$ total RNA was retro-transcribed with the RevertAid H Minus First Strand cDNA Synthesis Kit (Invitrogen). Expression levels of genes involved in the RNAi pathway were quantified by qPCR to confirm the results from the colorimetric microarray analysis. Primers for 11 reference genes and some target genes (Table S3) were used from the literature or newly designed with Primer3 (http://www.ncbi.nlm. nih.gov/tools/primer-blast/) using the default settings. For the RT-qPCR assays the SsoFast EvaGreen Supermix kit (Bio-Rad, Richmond, CA, USA) was used. Each $15 \mu \mathrm{L}$ reaction consisted of $7.5 \mu \mathrm{L}$ master mix, $0.2 \mu \mathrm{M}$ forward and $0.2 \mu \mathrm{M}$ reverse primers (Integrated DNA Technologies) and $1 \mu \mathrm{L}$ cDNA template using the CFX96 Real-Time PCR Detection System (Bio-Rad). The PCR program comprises an activation step of $1 \mathrm{~min}$ at $95^{\circ} \mathrm{C}$ and 40 cycles of a combined denaturation $(5 \mathrm{~s}$ at $\left.95^{\circ} \mathrm{C}\right)$ and annealing $\left(10 \mathrm{~s}\right.$ at $\left.60^{\circ} \mathrm{C}\right)$ step. At the end of this program a melt curve is generated by measuring fluorescence after each temperature increase of $0.5^{\circ} \mathrm{C}$ for $5 \mathrm{~s}$ over a range from $65^{\circ} \mathrm{C}$ to $95^{\circ} \mathrm{C}$. Primer efficiencies, $\mathrm{R} 2$ values and melt curves were calculated with CFX Manager software (Bio-Rad). Reference gene stability was analyzed with the geNormPLUS algorithm within the qBasePLUS environment (Biogazelle NV, Zwijnaarde, Belgium) with default settings. Differential gene expression of target genes was statistically analyzed using qBasePLUS by means of Mann-Whitney tests. Two-sided significance and correction for multiple testing were applied.

\section{RESULTS}

\section{Overview of differential gene expression from some target genes as revealed by microarray analysis}

The effect of natural viral infection on the gene expression of the different targets was determined by comparing the gene expression of naturally high virus infected (HVI) honeybees with that of low virus infected (LVI) honeybees. The expression levels were normalized using RPL8 as a reference gene. In Table 1 , the expression ratios from different immunity genes and genes involved in the RNAi antiviral response from HVI and LVI honeybees are given. As the dynamic range of the colorimetric array was low, we used this array as a first screening method to determine a set of differential expressed genes. Most of the immunity genes in the HVI individuals were diminished in expression levels compared to LVI individuals. The gene expression levels of most of the genes involved in the RNAi machinery were also decreased in the HVI group. The results of the screening with the micro-array, developed in-house and called BeeClinic, showed that high virus loads in honeybee are accompanied by suppression of the key components of the RNAi machinery.

\section{Reference gene selection for normalization of qRT-PCR data}

The reliability of the data of the microarray experiment was checked with qRT-PCR. To correct for experimental error, qPCR data require normalization against reference genes (Bustin etal. 2009). Thus, 11 reference genes were selected (Table S3) and their expression was quantified in HVI and LVI honeybees. The genes from most to least stably expressed across all conditions produced the following ranking (Fig. $\mathrm{S} 1 \mathrm{~A}$ ): $\quad$ eIF $>$ RPS5 $>$ $R P L 8>$ enolase $>M G S T>G A P D H>$ actine $>R P 49>-$ $T B P>R P L 13 a>R P S 18$. Another measure, the geNorm $V$-value, is useful for determining the optimal number of reference genes for data normalization (Fig. S1B): V2/3 $(0.214)$ - V3/4 (0.157) - V4/5 (0.200) - V5/6 (0.166) V6/7 (0.155) - V7/8 (0.151) - V8/9 (0.141). Setting the threshold to 0.15 , six genes should be included in the calculation of the normalization factors (Vandesompele etal. 2002; Hellemans et al. 2007).

\section{Validation of microarray data by qRT-PCR}

Microarray validation by qRT-PCR was performed with all genes involved in the RNAi machinery (Table 2). For four genes, Dicer-like (Dcr-like), Dicer1 (Dcr1), scavenger receptor class $C$ type I (SCR-C) and TAR RNA-binding protein $(T A R B P)$, we could show a significant decrease in expression in the HVI group. However, it is worthwhile to mention that all the other genes involved in the RNAi machinery showed also lower expression levels, which suggest a suppression of the entire RNAi machinery. Expression profiles obtained in the qRT-PCR experiment were similar to those of the microarray experiment, except that for Ago2 opposite expression levels were obtained.

\section{DISCUSSION}

Viruses are one of the major threats for honeybees and until now, more than 20 different viruses have been 
Table 1 Expression ratios from different immunity genes and genes involved in the RNAi anti-viral response from HVI versus LVI honeybees

\begin{tabular}{|c|c|c|c|c|}
\hline ID & Expression change & $\log \mathrm{FC}$ & $P$ & adj. $P$ \\
\hline \multicolumn{5}{|l|}{ Immunity-related genes } \\
\hline Myd88 & $\downarrow$ & -0.2138400696 & 0.0162492275 & 0.3668728645 \\
\hline Defensin 2 & $\downarrow$ & -0.3123921013 & 0.1115784119 & 0.5895526928 \\
\hline$L y s-2$ & $\downarrow$ & -0.0956489282 & 0.1697480242 & 0.6378612144 \\
\hline Apisimin & $\uparrow$ & 0.3539765077 & 0.2004993572 & 0.6501039765 \\
\hline pGRP9710 & $\downarrow$ & -0.1345275462 & 0.2565079163 & 0.6619780418 \\
\hline Apidaecin & $\uparrow$ & 0.1661452114 & 0.3721433519 & 0.7203635361 \\
\hline Hopscotch & $\downarrow$ & -0.1498095232 & 0.3467253496 & 0.7203635361 \\
\hline Kenny & $\downarrow$ & -0.1356421311 & 0.3535630254 & 0.7203635361 \\
\hline pGRPLC710R & $\downarrow$ & -0.1127950494 & 0.3787671635 & 0.7203635361 \\
\hline Basket & $\uparrow$ & 0.0713399039 & 0.4646187816 & 0.7757623218 \\
\hline BglucA & $\downarrow$ & -0.1088962985 & 0.5147128215 & 0.7757623218 \\
\hline Cactus-1 & $\downarrow$ & -0.1090418077 & 0.4819047884 & 0.7757623218 \\
\hline Domeless & $\downarrow$ & -0.1319723889 & 0.5308397298 & 0.7757623218 \\
\hline Dorsal-1 & $\downarrow$ & -0.0693316542 & 0.462003764 & 0.7757623218 \\
\hline$L y s-1$ & $\downarrow$ & -0.0498326995 & 0.500786236 & 0.7757623218 \\
\hline Perseph & $\downarrow$ & -0.1270461796 & 0.4807258399 & 0.7757623218 \\
\hline pGRPSC2505 & $\downarrow$ & -0.1153286919 & 0.4836842337 & 0.7757623218 \\
\hline PPOact & $\downarrow$ & -0.1171048375 & 0.4843593599 & 0.7757623218 \\
\hline Spaetzle & $\downarrow$ & -0.0780592746 & 0.5301151514 & 0.7757623218 \\
\hline Hemipterous & $\downarrow$ & -0.1192836181 & 0.573967886 & 0.7788792605 \\
\hline$T a b$ & $\downarrow$ & -0.0622960477 & 0.5808615216 & 0.7788792605 \\
\hline Dredd & $\downarrow$ & -0.0756838627 & 0.595243977 & 0.7863099449 \\
\hline Cactus-2 & $\downarrow$ & -0.0620173169 & 0.6099163588 & 0.7912324918 \\
\hline Relish & $\downarrow$ & -0.0470041476 & 0.6137597833 & 0.7912324918 \\
\hline Dscam-37 & $\uparrow$ & 0.0466894594 & 0.6451372703 & 0.821454886 \\
\hline TEPA & $\downarrow$ & -0.0411024341 & 0.7394139642 & 0.8790810463 \\
\hline$L y s-3 I$ & $\downarrow$ & -0.041268914 & 0.800169192 & 0.9306315603 \\
\hline Abaecin & $\uparrow$ & 0.0396080372 & 0.809465864 & 0.9313209403 \\
\hline Dscam & $\downarrow$ & -0.0233648515 & 0.8244768753 & 0.938500273 \\
\hline EGFlikeA = Eater & $\downarrow$ & -0.0235614594 & 0.8475223432 & 0.9530921315 \\
\hline Defensin 1 & $\uparrow$ & 0.0241697618 & 0.9136585636 & 0.961897805 \\
\hline Hymenopt & $\uparrow$ & 0.0182766292 & 0.9158476679 & 0.961897805 \\
\hline Imd & $\downarrow$ & -0.010126515 & 0.9208859191 & 0.961897805 \\
\hline pGRPSC4300 & $\downarrow$ & -0.0189983869 & 0.906768034 & 0.961897805 \\
\hline TEP7 & $\uparrow$ & 0.0257638135 & 0.8829159556 & 0.961897805 \\
\hline AmPPO & $\uparrow$ & 0.0058257697 & 0.9646463432 & 0.9764964622 \\
\hline Dorsal-2 & $\uparrow$ & 0.0078146459 & 0.9508312236 & 0.9764964622 \\
\hline Tak1 & $\uparrow$ & 0.0034580313 & 0.9673703271 & 0.9764964622 \\
\hline Toll & $\downarrow$ & -0.0053407889 & 0.9790570823 & 0.9790570823 \\
\hline \multicolumn{5}{|l|}{ RNAi-related genes } \\
\hline Aubergine & $\downarrow$ & -0.4750271686 & 0.0054629947 & 0.3668728645 \\
\hline Dicer2 & $\downarrow$ & -0.301630163 & 0.0279449642 & 0.3858134763 \\
\hline Dicer1 & $\downarrow$ & -0.2740781564 & 0.0503066365 & 0.4485675084 \\
\hline Ago2 & $\uparrow$ & 0.3869129667 & 0.1122432583 & 0.5895526928 \\
\hline Dicer2 & $\downarrow$ & -0.3120556635 & 0.1154024703 & 0.5895526928 \\
\hline$T R B P 2$ & $\downarrow$ & -0.2775895973 & 0.1183784066 & 0.5895526928 \\
\hline Sid-I & $\downarrow$ & -0.3161457773 & 0.0781852908 & 0.5895526928 \\
\hline Ago1 & $\downarrow$ & -0.2063470201 & 0.1907622324 & 0.6378612144 \\
\hline Pasha homologue & $\downarrow$ & -0.2466573824 & 0.1664867898 & 0.6378612144 \\
\hline$S R C-C$ & $\downarrow$ & -0.2321851438 & 0.1631458939 & 0.6378612144 \\
\hline$T A R B P$ & $\downarrow$ & -0.1562478829 & 0.2411147007 & 0.6619780418 \\
\hline Drosha homologue & $\downarrow$ & -0.1892093027 & 0.3266593987 & 0.7203635361 \\
\hline Dicer2 & $\uparrow$ & 0.1182388252 & 0.374073057 & 0.7203635361 \\
\hline
\end{tabular}


L. De Smet et al.

TABLE 1. (Continued)

\begin{tabular}{|c|c|c|c|c|}
\hline ID & Expression change & $\log \mathrm{FC}$ & $P$ & adj. $P$ \\
\hline Ago3 & $\uparrow$ & 0.1257475242 & 0.4929145669 & 0.7757623218 \\
\hline Argonaute2 & $\downarrow$ & -0.1030457037 & 0.4961182051 & 0.7757623218 \\
\hline Stan & $\uparrow$ & 0.0183886426 & 0.9168821052 & 0.961897805 \\
\hline \multicolumn{5}{|l|}{ Other marker genes } \\
\hline$m$ For & $\downarrow$ & -0.3955084084 & 0.0076561118 & 0.3668728645 \\
\hline$I R S$ & $\downarrow$ & -0.3000527674 & 0.0205723102 & 0.3668728645 \\
\hline Juvenile hormone esterase & $\downarrow$ & -0.3813597363 & 0.0205536632 & 0.3668728645 \\
\hline TOR & $\downarrow$ & -0.3346384267 & 0.0118364791 & 0.3668728645 \\
\hline InR-2 (daf-2) & $\downarrow$ & -0.4080606105 & 0.0311102591 & 0.3858134763 \\
\hline Glucose dehydrogenase & $\downarrow$ & -0.3341599151 & 0.0464487427 & 0.4485675084 \\
\hline$R p d 3$ & $\downarrow$ & -0.3391419852 & 0.0472851649 & 0.4485675084 \\
\hline$\alpha$ Mannosidase I & $\downarrow$ & -0.2116531287 & 0.1267262798 & 0.5895526928 \\
\hline Hemolectin & $\downarrow$ & -0.5304977143 & 0.0869008049 & 0.5895526928 \\
\hline Malvolio & $\downarrow$ & -0.2652851109 & 0.0795292457 & 0.5895526928 \\
\hline Painless & $\downarrow$ & -0.2699401084 & 0.1177320908 & 0.5895526928 \\
\hline Sluggish A & $\downarrow$ & -0.2413689417 & 0.1071555905 & 0.5895526928 \\
\hline$d \operatorname{Sir} 2$ & $\downarrow$ & -0.2532656245 & 0.1820991154 & 0.6378612144 \\
\hline Pheromone biosynthesis-activating neuropeptide & $\uparrow$ & 0.1930591825 & 0.185453636 & 0.6378612144 \\
\hline Stretchin-Mlck & $\downarrow$ & -0.2288870438 & 0.1904190464 & 0.6378612144 \\
\hline Thioredoxin reductase-1 & $\downarrow$ & -0.1945863754 & 0.1697007671 & 0.6378612144 \\
\hline Hexamerin $70 \mathrm{a}$ & $\downarrow$ & -0.1758682431 & 0.2087699864 & 0.6570114278 \\
\hline Catalase & $\downarrow$ & -0.2030069205 & 0.2227440116 & 0.6605118885 \\
\hline$\alpha$ glucosidase $(\mathrm{Hbg} 1)$ & $\downarrow$ & -0.1345532464 & 0.2598418482 & 0.6619780418 \\
\hline Hairy & $\downarrow$ & -0.1229296117 & 0.251853108 & 0.6619780418 \\
\hline Armadillo & $\uparrow$ & 0.118474724 & 0.2729416256 & 0.6791803242 \\
\hline Corticotropin releasing hormone binding protein & $\downarrow$ & -0.1295469453 & 0.3400031205 & 0.7203635361 \\
\hline Cytochrome P450 monooxygenase (Cyp4g11) & $\uparrow$ & 0.104544683 & 0.3322755967 & 0.7203635361 \\
\hline Facilitated trehalose transporter Tret1-like & $\downarrow$ & -0.1534007353 & 0.3492074685 & 0.7203635361 \\
\hline Poly U binding factor $68 \mathrm{kD}$ & $\downarrow$ & -0.1272054412 & 0.3837450613 & 0.7203635361 \\
\hline Prophenoloxidase & $\downarrow$ & -0.1244017237 & 0.3040654398 & 0.7203635361 \\
\hline Sugarless & $\downarrow$ & -0.0964444261 & 0.3732255014 & 0.7203635361 \\
\hline Futsch & $\downarrow$ & -0.100580448 & 0.5365085216 & 0.7757623218 \\
\hline Superoxide dismutase & $\uparrow$ & 0.1092696452 & 0.5078646115 & 0.7757623218 \\
\hline Trehalose transporter 1 & $\downarrow$ & -0.102442541 & 0.5266524472 & 0.7757623218 \\
\hline EGFR & $\uparrow$ & 0.0646858736 & 0.582339634 & 0.7788792605 \\
\hline $\mathrm{Na}$ pump subunit & $\downarrow$ & -0.0813559192 & 0.5605051046 & 0.7788792605 \\
\hline Vitellogenin & $\downarrow$ & -0.1085209875 & 0.5725400384 & 0.7788792605 \\
\hline Dopamine receptor, D1 & $\downarrow$ & -0.0731462832 & 0.6602347682 & 0.821454886 \\
\hline dPGC-1/spargel & $\downarrow$ & -0.0694665547 & 0.6942751682 & 0.8346903708 \\
\hline Foxo & $\uparrow$ & 0.0638067343 & 0.7700727751 & 0.9054701861 \\
\hline Transferrin & $\uparrow$ & 0.0249853511 & 0.8551106974 & 0.9530921315 \\
\hline
\end{tabular}

HVI, high virus infected; LVI, low virus infected; log FC, $\log$ (fold expression); adj. $P$, adjusted $P$-value calculated using the BH method (Smyth 2004$) ; \uparrow$, unregulated; $\downarrow$, downregulated.

discovered (Evans \& Schwarz 2011). Viruses and their hosts are in continuous competition in which viral counter-deface mechanisms drive the adaptive evolution of host immune genes, which in turn results in counteradaptations of the viral immune antagonists (Daugherty \& Malik 2012).

Most honeybee viruses are +ssRNA viruses. These viruses produce dsRNA as replication intermediates. The dsRNA is often a molecular signature of virus infection and as such is a trigger for a range of host responses, including RNA interference. Viral dsRNA may feed into the RNAi machinery to restrict virus replication (Bronkhorst \& van Rij 2014). Several mechanisms were predicted by which virus infections can interfere with RNAi (Swevers etal. 2013). These include the expression of VSRs, accumulation of large amounts of viral RNAs and small RNAs that will overflow the RNAi machinery, modulation of expression of host miRNAs and expression of viral miRNAs, and induction of a general antiviral state in infected insects. 
Table 2 Quantitative polymerase chain reaction validation results

\begin{tabular}{lll}
\hline Gene & Ratio & \multicolumn{1}{c}{$P$-value } \\
\hline Ago & 0.477 & 0.075 \\
Ago2 & 0.160 & 0.094 \\
Ago3 & 0.552 & 0.084 \\
Aub & 0.443 & 0.316 \\
Dicer2 & 0.264 & $4.615 \times 10^{-2 *}$ \\
Dicer1 & 0.529 & $4.615 \times 10^{-2} *$ \\
Drosha homologue & 0.771 & 0.399 \\
SCR-C & 0.278 & $4.615 \times 10^{-2 *}$ \\
TARBP & 0.423 & $4.930 \times 10^{-2} *$ \\
\hline
\end{tabular}

* Significantly different.

Different studies in honeybees could already show the existence of virus-specific siRNA on viral infection but until now evidence is lacking about the exact mechanism (Flenniken \& Andino 2013; Ryabov etal. 2014). Several transcriptome studies could not show significant changes in gene expression of key components, such as Dicer and Argonaute, of the RNAi response (Flenniken \& Andino 2013; Ryabov etal. 2014). These studies were mostly performed with experimentally infected honeybees. The advantage of working with experimentally infected honeybees is that the genetic background of all the bees is the same. On the other hand, mostly just emerging bees are artificially infected, which means that the immunity response is determined on a relatively short period. When working with naturally infected honeybees the infections occurred naturally and the copy number of the different viruses will be relatively low; moreover bees benefit from social immune behaviors (i.e. grooming and behavioral fever), which may reduce colony pathogen burden (Evans \& Spivak 2010) and will not be present in caged honeybees. Another advantage of working with naturally infected honeybees is that in this way the natural response of the honeybee in its natural environment can be studied. We selected natural HVI honeybees and performed an inhouse colorimetric array where all key components of the RNAi response were targeted. Although the dynamic range of the colorimetric array is low and no significant gene expression could be shown, we were able to show changed expression levels of all genes involved in the RNAi machinery. This screening method is meant for a rapid screening of changed expression levels from a selected set of targets and show trends in the expression levels of a certain set of selected targets. The expression levels of most targets were lower in highly infected honeybees compared to LVI bees. Validation with qPCR showed that the genes encoding two Dicer proteins, SCR-C and TARBP, were expressed significantly lower. It seems that the viruses are able to highjack the RNAi machinery although the exact mechanism remains unclear. Emerging evidence indicates that some animal viruses alter the expression of components of the RNAi machinery. Dicer mRNA and protein expression levels were also downregulated in mammalian cells infected with influenza A virus or dengue virus (Matskevich \& Moelling 2007). Dicer, TARBP and SCR-C are all proteins that can be targeted by VSRs and it has been shown that the mRNA levels can also be modulated through viral infection. Viral suppressors do not have common sequence motifs and act at different steps of the RNAi pathway. VSRs can interfere with the activity of Dicer, which leads to suppression of siRNA biogenesis (Qi etal. 2012). TARBP is a co-factor of Dicer that functions as part of the RISC complex and was shown to be a target of VSRs encoded by HIV-1 (Bennasser etal. 2006). The mRNA levels for both targets were significantly downregulated in our study, which suggests the existence of VSR expression in honeybee viruses. The mRNA levels of SCR-C were also significantly lower in highly infected honeybees. This is a receptor that is important for dsRNA uptake. In Drosophila, exogenous dsRNA enters the RNAi pathway by scavenger receptor-mediated endocytosis and it was shown that RNAi uptake is essential in the process of antiviral defense. However until now there is no evidence that SCR-C is a target for virus-encoded VSRs.

Our results suggest that honeybee viruses are able to usurp the host RNAi machinery by means of suppression genes of the key components. The recent discovery that honeybee viruses of the ABPV complex encode VSRs located upstream of a DVEXNPGP motif located in the N-terminal region of ORF-1 (Chen etal. 2014) strongly supports this hypothesis.

In conclusion, we analyzed the differential expressed genes in honeybees with a high virus load compared to honeybees with a low virus load. The microarray developed in-house, called BeeClinic, showed that the genes involved in the RNAi pathway are mostly suppressed, which was confirmed by qRT-PCR. The suppression of the different key RNAi components (Dcr2, SCR-C and TARBP) supports the hypothesis that honeybee viruses are able to suppress the host RNAi machinery in order to infect and replicate within their host.

\section{ACKNOWLEDGMENTS}

This work was supported by the European Commission through the 7th framework collaborative project Bees in Europe and the Decline of Honeybee Colonies (BEE DOC; EU contract number: FP7-KBBE-2009-3 244956 CP-FP) and by the Fund Research Foundation of Flanders (FWO-Vlaanderen G.0628.11). 


\section{REFERENCES}

Alaux C, Dantec C, Parrinello H, Le Conte Y (2011) Nutrigenomics in honey bees: digital gene expression analysis of pollen's nutritive effects on healthy and varroaparasitized bees. BMC Genomics 12, Article ID 496. DOI:10.1186/1471-2164-12-496

Behrens D, Huang Q, Gessner C etal. (2011) Three QTL in the honey bee Apis mellifera L. suppress reproduction of the parasitic mite Varroa destructor. Ecology and Evolution 1, 451-458.

Bennasser Y, Yeung ML, Jeang KT (2006) HIV-1 TAR RNA subverts RNA interference in transfected cells through sequestration of TAR RNA-binding protein, TRBP. Journal of Biological Chemistry 281, 27674-27678.

Blair CD (2011) Mosquito RNAi is the major innate immune pathway controlling arbovirus infection and transmission. Future Microbiology 6, 265-277.

Bronkhorst AW, van Rij RP (2014) The long and short of antiviral defense: small RNA-based immunity in insects. Current Opinion in Virology 7, 19-28.

Bustin SA, Benes V, Garson JA et al. (2009) The MIQE guidelines: minimum information for publication of quantitative real-time PCR experiments. Clinical Chemistry 55, 611-622.

Campbell CL, Keene KM, Brackney DE etal. (2008) Aedes aegypti uses RNA interference in defense against Sindbis virus infection. BMC Microbiology 8, Article ID 47. DOI:10.1186/1471-2180-8-47

Chan QWT, Melathopoulos AP, Pernal SF, Foster LJ (2009) The innate immune and systemic response in honey bees to a bacterial pathogen, Paenibacillus larvae. BMC Genomics 10, Article ID 387. DOI:10.1186/1471-2164-10-387

Chejanovsky N, Ophir R, Schwager MS, Slabezki Y, Grossman S, Cox-Foster D (2014) Characterization of viral siRNA populations in honey bee colony collapse disorder. Virology 454-455, 176-183.

Chen Y (2011) Viruses and viral diseases of the honey bee, Apis mellifera. In: Liu T, Kang L (eds) Recent Advances in Entomological Research, pp 105-120. Springer, BerlinHeidelberg.

Chen YP, Siede R (2007) Honey bee viruses. Advances in Virus Research 70, 33-80.

Chen YP, Pettis JS, Corona M et al. (2014) Israeli acute paralysis virus: epidemiology, pathogenesis and implications for honey bee health. PLoS Pathogens 10, Article ID e1004261. DOI:10.1371/journal.ppat.1004261

Chinnappan M, Singh AK, Kakumani PK etal. (2014) Key elements of the RNAi pathway are regulated by hepatitis B virus replication and $\mathrm{HBx}$ acts as a viral suppressor of RNA silencing. Biochemical Journal 462, 347-358.

Daugherty MD, Malik HS (2012) Rules of engagement: molecular insights from host-virus arms races. Annual Review of Genetics 46, 677-700.

de Miranda JR, Gauthier L, Ribiere LM, Chen YP (2012) Honey bee viruses and their effect on bee and colony health. In: Sammataro D, Yoder J (eds) Honey Bee Colony Health:
Challenges and Sustainable Solutions, pp 71-102. CRC Press, Boca Raton, FL.

Desai SD, Eu YJ, Whyard S, Currie RW (2012) Reduction in deformed wing virus infection in larval and adult honey bees (Apis mellifera L.) by double-stranded RNA ingestion. Insect Molecular Biology 21, 446-455.

De Smet L, Ravoet J, de Miranda JR etal. (2012) BeeDoctor, a versatile MLPA-based diagnostic tool for screening bee viruses. PLoS One 7, Article ID e47953. DOI:10.1371/ journal.pone.0047953

Ding SW (2010) RNA-based antiviral immunity. Nature Reviews Immunology 10, 632-644.

Dussaubat C, Brunet JL, Higes M etal. (2012) Gut pathology and responses to the microsporidium Nosema ceranae in the honey bee Apis mellifera. PLoS One 7, Article ID e37017. DOI:10.1371/journal.pone.0037017

Elbashir SM, Harborth J, Lendeckel W, Yalcin A, Weber K, Tuschl T (2001) Duplexes of 21-nucleotide RNAs mediate RNA interference in cultured mammalian cells. Nature 41, 494-498.

Evans JD (2006) Beepath: an ordered quantitative-PCR array for exploring honey bee immunity and disease. Journal of Invertebrate Pathology 93, 135-139.

Evans JD, Schwarz RS (2011) Bees brought to their knees: microbes affecting honey bee health. Trends in Microbiology 19, 614-620.

Evans JD, Spivak M (2010) Socialized medicine: individual and communal disease barriers in honey bees. Journal of Invertebrate Pathology 103(Suppl 1), S62-S72.

Evans JD, Aronstein K, Chen YP etal. (2006) Immune pathways and defence mechanisms in honey bees Apis mellifera. Insect Molecular Biology 15, 645-656.

Flenniken ML, Andino R (2013) Non-specific dsRNA-mediated antiviral response in the honey bee. PLoS One 8, Article ID e77263. DOI:10.1371/journal.pone.0077263

Goic B, Vodovar N, Mondotte JA et al. (2013) RNA-mediated interference and reverse transcription control the persistence of RNA viruses in the insect model Drosophila. Nature Immunology 14, 396-403.

Hammond SM, Boettcher S, Caudy AA, Kobayashi R, Hannon GJ (2001) Argonaute2, a link between genetic and biochemical analyses of RNAi. Science 293, 1146-1150.

Hellemans J, Mortier G, De Paepe A, Speleman F, Vandesompele $\mathrm{J}$ (2007) qBase relative quantification framework and software for management and automated analysis of realtime quantitative PCR data. Genome Biology 8, Articel ID R19. DOI: 10.1186/gb-2007-8-2-r19

Huang Q, Kryger P, Le Conte Y, Moritz RF (2012) Survival and immune response of drones of a Nosemosis tolerant honey bee strain towards N.ceranae infections. Journal of Invertebrate Pathology 109, 297-302.

Johnson RM, Evans JD, Robinson GE, Berenbaum MR (2009) Changes in transcript abundance relating to colony collapse disorder in honey bees (Apis mellifera). Proceedings of the National Academy of Sciences of the United States of America 106, 14 790-14795.

Karlikow M, Goic B, Saleh MC (2014) RNAi and antiviral defense in Drosophila: Setting up a systemic immune 
response. Developmental and Comparative Immunology 42, 85-92.

Kemp C, Imler JL (2009) Antiviral immunity in Drosophila. Current Opinion in Immunology 21, 3-9.

Lemaitre B, Hoffmann J (2007) The host defense of Drosophila melanogaster. Annual Review of Immunology 25, 697-743.

Li F, Ding SW (2006) Virus counterdefense: Diverse strategies for evading the RNA-silencing immunity. Annual Review of Microbiology 60, 503-531.

Li JL, Cornman RS, Evans JD etal. (2014) Systemic spread and propagation of a plant-pathogenic virus in European honeybees, Apis mellifera. MBio 5, Article ID e00898-13. DOI:10.1128/mBio.00898-13

Liu X, Zhang Y, Yan X, Han R (2010) Prevention of Chinese sacbrood virus infection in Apis cerana using RNA interference. Current Microbiology 61, 422-428.

Maori E, Paldi N, Shafir S et al. (2009) IAPV, a bee-affecting virus associated with Colony Collapse Disorder can be silenced by dsRNA ingestion. Insect Molecular Biology 18, 55-60.

Matskevich AA, Moelling K (2007) Dicer is involved in protection against influenza A virus infection. Journal of General Virology 88, 2627-2635.

McMenamin AJ, Genersch E (2015) Honey bee colony losses and associated viruses. Current Opinion in Insect Science 8, 121-129.

Nayak A, Berry B, Tassetto M et al. (2010) Cricket paralysis virus antagonizes Argonaute 2 to modulate antiviral defense in Drosophila. Nature Structure \& Molecular Biology 17, 547-554.

Niu J, Meeus I, Cappelle K, Piot N, Smagghe G (2015) The immune response of the small interfering RNA pathway in the defense against bee viruses. Current Opinion in Insect Science 6, 22-27.

O’Neal ST, Samuel GH, Adelman ZN, Myles KM (2014) Mosquito-borne viruses and suppressors of invertebrate antiviral RNA silencing. Viruses 6, 4314-4331.

Piot N, Snoeck S, Vanlede M, Smagghe G, Meeus I (2015) The effect of oral administration of dsRNA on viral replication and mortality in Bombus terrestris. Viruses 7, 3172-3185.

Qi N, Zhang L, Qiu Y et al. (2012) Targeting of dicer-2 and RNA by a viral RNA silencing suppressor in Drosophila cells. Journal of Virology 86, 5763-5773.

Ravoet J, Maharramov J, Meeus I etal. (2013) Comprehensive bee pathogen screening in Belgium reveals Crithidia mellificae as a new contributory factor to winter mortality. PLoS One 8, Article ID e72443. DOI:10.1371/journal. pone. 0072443

Ritchie ME, Phipson B, Wu D etal. (2015) limma powers differential expression analyses for RNA sequencing and microarray studies. Nucleic Acids Research 43, Article ID e47. DOI:10.1093/nar/gkv007
Runckel C, Flenniken ML, Engel JC etal. (2011) Temporal analysis of the honey bee microbiome reveals four novel viruses and seasonal prevalence of known viruses, Nosema, and Crithidia. PLoS One 6, Article ID e20656. DOI:10.1371/journal.pone.0020656

Ryabov EV, Wood GR, Fannon JM et al. (2014) A virulent strain of deformed wing virus (DWV) of honeybees (Apis mellifera) prevails after Varroa destructor-mediated, or in vitro, transmission. PLoS Pathogens 10, Article ID e1004230. DOI:10.1371/journal.ppat.1004230

Saleh MC, Tassetto M, van Rij RP etal. (2009) Antiviral immunity in Drosophila requires systemic RNA interference spread. Nature 458, 346-350.

Schwarz RS, Evans JD (2013) Single and mixed-species trypanosome and microsporidia infections elicit distinct, ephemeral cellular and humoral immune responses in honey bees. Developmental and Comparative Immunology 40, 300-310.

Smyth GK (2004) Linear models and empirical Bayes methods for assessing differential expression in microarray experiments. Statistical Applications in Genetics and Molecular Biology 3, 1-25.

Swevers L, Vanden Broeck J, Smagghe G (2013) The possible impact of persistent virus infection on the function of the RNAi machinery in insects: a hypothesis. Frontiers in Physiology 4, Article ID 319. DOI:10.3389/ fphys.2013.00319

Vandesompele J, De Preter K, Pattyn F etal. (2002) Accurate normalization of real-time quantitative RT-PCR data by geometric averaging of multiple internal control genes. Genome Biology 3, 1-12.

\section{SUPPORTING INFORMATION}

Additional Supporting Information may be found in the online version of this article at the publisher's web-site:

Table S1 Virus state of the different samples used in this study.

Table S2 Locus names, gene function or marker, oligo sequence and accession numbers for genes.

Table S3 List of PCR primers used in this study for qPCR.

Figure S1 Average expression stability (A) and determination of the optimal number (B) of reference targets with geNormPLUS. 